\title{
Erratum: Spatially resolved and time-resolved imaging of transport of indirect excitons in high magnetic fields [Phys. Rev. B 95, 235308 (2017)]
}

C. J. Dorow, M. W. Hasling, E. V. Calman, L. V. Butov, J. Wilkes, K. L. Campman, and A. C. Gossard

Q (Received 30 January 2019; published 7 February 2019)

DOI: 10.1103/PhysRevB.99.079902

The units of the $y$ axis for Figs. 3(c) and 7(b) should be " $\mu \mathrm{m}^{2} / \mathrm{ns}^{\prime}$ instead of " $\mathrm{cm}^{2} / \mathrm{s}$." The text and conclusion of the paper remain intact. 